\title{
Current distribution in a parallel set of conducting strips
}

\author{
T. Ulicevic, J.M.B. Kroot, S.J.L. van Eijndhoven, A.A.F. van de Ven \\ Eindhoven University of Technology \\ P.O.Box 513; 5600 MB Eindhoven, The Netherlands
}

February 19, 2004

date: February 19, 2004

\begin{abstract}
The current distribution in a parallel set of thin conducting sheets due to an external applied source is investigated. All sheets are placed in one plane. The source, and all excited fields, are time harmonic. The frequency is low enough to allow for an electro quasi-static approximation (neglecting the displacement current). The conducting sheets are infinitely long and the current is uniform in the longitudinal direction of the sheets. The sheets have a thin rectangular cross-section, so thin that the current can be assumed uniform in the thickness-direction. Hence, the current distribution only depends on the transverse coordinate. Due to the mutual induction between the sheets, the current distribution over the width of the cross-section becomes non-uniform: it accumulates at the edges of the sheets. It is especially this so-called edge-effect, and its dependence on the applied frequency and the distances between the sheets, that is the aim of this investigation. From the Maxwell equations, a set of integral equations for the current distribution in the sheets is derived. These integral equations are solved, as far as possible by analytical means, by writing the current distribution in each sheet as a series of Legendre polynomials. The general method is worked out for $N(N \geq 1)$ sheets, but explicit results are presented for $N=1$ and $N=3$. It turns out that the edge-effect becomes stronger for increasing frequencies. For this solution, only a very restricted number of Legendre polynomials is needed.
\end{abstract}

\section{Introduction}

Magnetic Resonance Imaging (MRI) is a non-destructive way of scanning a human body for medical diagnostics, but it can also be used in other applications (e.g. for the scanning of the drying process in clay tiles). A general description of the process of MRI is presented in the book by Vlaardingerbroek and Den Boer [1]. In an MRI scanner, the so-called main magnet produces a homogeneous static magnetic field. To enable the spatial information 
of MR images, the magnetic field strength must show variation in space in a controllable manner. Such variation is provided by gradient coils, producing linearly varying magnetic fields. These gradient fields must be as linear as possible in the centre of the scanner with respect to spatial variables.

The configuration of the gradient coil constitutes of a circular cylinder covered by a given pattern of very thin conductors. The conducting sheets are of copper, having a finite resistance. The sheet has a finite width and spirals in its length direction along the cylindrical surface. The coil is excited by a given dynamic input current.

One of the major problems in the use of gradient coils is the interaction of the rapidly fluctuating fields with other conducting structures in the MRI scanner including other parts of the gradient coils. The interaction due to mutual induction causes non-uniform current distributions in the sheets. It turns out that at sufficiently high frequencies the current accumulates at the edges of the sheets. This so-called edge-effect causes perturbations on the expected gradient field and, consequently, in the NMR-picture ("blurring"), and should therefore be avoided as much as possible.

Due to the complexity of the geometry of the gradient coil we have simplified the problem considerably: the configuration we consider here consists of a set of $N(N \geq 1)$ parallel conducting sheets of a narrow rectangular cross-section and of infinite length. We shall show that this simplified configuration already reveals when, i.e. for which frequencies, edge-effects occur, and how they are affected by the distance between the sheets. Moreover, the methods developed here can, and will, also be applied to more general structures such as sets of parallel conducting rings on a cylinderical surface.

The longitudinal direction of the strips is taken in the $z$-direction, while the $x$-axis is in the width-direction and the $y$-axis in the thickness-direction; see Figure 1. All sheets have thickness $2 h$, but they may have different widths: the $p^{t h}$ sheet $(p \in[1, N])$ having width $2 d_{p}=\left(b_{p}-a_{p}\right)$. The thickness of the sheets is always much smaller than their width. In the next section, we shall show that we may assume the sheets infinitely thin in $y$-direction. We call such an infinitely thin sheet a strip. All strips are lying in one plane. These strips occupy the surface $S$ in Euclidean space $\mathbb{R}^{3}$, described in Cartesian coordinates by

$$
\mathcal{S}=\bigcup_{p=1}^{N} \mathcal{S}_{p}, \quad \mathcal{S}_{p}=\left\{(x, 0, z) \in \mathbb{R}^{3} \mid \mathrm{x} \in\left[\mathrm{a}_{\mathrm{p}}, \mathrm{b}_{\mathrm{p}}\right], \mathrm{a}_{\mathrm{p}}<\mathrm{b}_{\mathrm{p}}\right\}
$$

Through the sheets, an electric current is flowing in longitudinal or $z$-direction. The total current through a sheet, or through a group of sheets, is prescribed; the precise distribution of this current is the main unknown of this article. Its determination, by as far as possible analytical means, is our first aim. The sheets are good conductors and the current is timeharmonic. The frequency is small enough to allow for an electro quasi-static approximation for the governing Maxwell equations. In this case, the current is uniform in the $z$-direction.

The present paper is based on a postgraduate project related to the design of gradient coils for MRI-scanners, performed at Philips Medical Systems, Best, The Netherlands, by the first author [2]. This work is somewhat generalized here, by including the possibility of arbitrarily prescribing the source currents on (groups of) strips. In [3] and [4], the second author has further developed the theory to more general structures such as sets of rings or sets of rings combined with islands (having the shape of a part of a ring). In the latter structures, besides edge-effects, also self-eddies will occur. The design of gradient coils is an important subject in the development of MRI-scanners. Approaches using stream functions to determine optimal surface structures are reported by Peeren [5], and Tomasi [6]. In [5], 


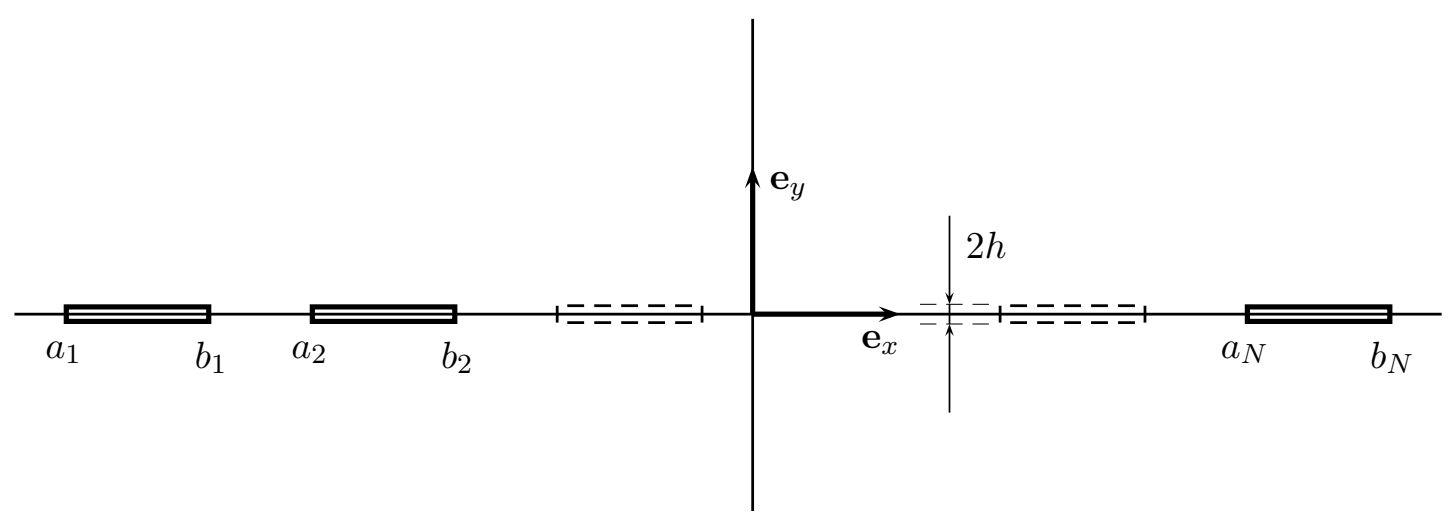

Figure 1: Configuration of a system of $N$ infinitely long parallel sheets; cross-section in $x$-y-plane.

the optimal structure is related to a minimization of the total energy. In this approach, the edge-effects are not taken into account. In [6], the current distribution is discretised by use of 1-dimensional wires. In the mathematical description of dynamic current distributions through strip-like structures, aspects as skin depth, see Landau and Lifshitz [7], and edgeeffects play an important role. Besides in gradient coils, strip-like structures also appear as striplines in electromagnetic transmission lines used for the excitation of antennas, see e.g. Collin [8]. However, in contrast to the (quasi-static) radio-frequency range in which gradient coils act (less than $10^{3} \mathrm{~Hz}$ ), the frequencies for antenna systems are very high $\left(\approx 10^{9} \mathrm{~Hz}\right)$, and in the latter case the strips can be modelled as perfectly conducting. Whether a conducting strip, dependent on the range of applied frequencies, can be considered as infinitely thin and/or perfectly conducting, is indicated by Bekers et al. [9]. In a paper by Genenko et al. [10], the reduction of edge currents due to magnetic shielding is simulated. Magnetic shielding is also considered in a paper by Forbes et al. [11], in which current distributions, inclusive eddy currents, in MRI-probes are computed. In most of the aforementioned papers, results are obtained purely by numerical simulations, whereas in the present paper we aim at an, as far as possible, analytical solution.

In Section 2, we will derive an integral equation for the current distribution in the sheets by applying the electromagnetic equations to the 3-dimensional space containing the strips. The situation for one strip will be analysed in Section 3, while that for $N$ strips will be worked out in Section 4. Solutions and numerical results will be shown in Section 5. Section 6 will present a survey of the conclusions and some recommendations for extending the analysis to more realistic models for gradient coils.

\section{Electromagnetic equations}

The basic equations for our problem are the well-known Maxwell equations. Both the sheets and the external region are assumed non-polarizable and non-magnetizable. Moreover, in the conducting sheets Ohm's law holds. We consider only time harmonic electromagnetic fields, i.e. fields that uniformly contain the term $e^{-i \omega t}, \omega \in \mathbb{R}^{+}$. Without further comments, this term will be omitted in our equations: fields are only space dependent and all time derivatives 
in the Maxwell equations are replaced by the factor $-i \omega$. We assume that the frequency range is restricted, in so far that an electro quasi-static approximation may be applied, implying that the displacement current (i.e. the term $\partial \mathbf{D} / \partial t$ in Ampere's law) and the instationary term $\partial \rho / \partial t$ in the law of conservation of charge may be neglected. The result of the latter neglect is a divergence-free current in the sheets (i.e. $\nabla \cdot \mathbf{J}=0$ ).

We denote by $\mathbf{H}$ the magnetic field strength, by $\mathbf{E}$ the electric field strength, and by $\mathbf{J}$ the current density, satisfying Ohm's law in the sheets. This leaves us with the following equations for the unknowns $\mathbf{E}(\mathbf{x}), \mathbf{H}(\mathbf{x})$ and $\mathbf{J}(\mathbf{x})$ :

$$
\begin{gathered}
\nabla \times \mathbf{E}=i \omega \mu \mathbf{H}, \quad \nabla \cdot \mathbf{E}=0, \\
\nabla \times \mathbf{H}=\mathbf{J}, \quad \nabla \cdot \mathbf{H}=0,
\end{gathered}
$$

and, moreover, in the sheets

$$
\nabla \cdot \mathbf{J}=0, \quad \mathbf{J}=\sigma \mathbf{E} .
$$

Here, $\mu=\mu_{0}$ and $\sigma$ are the permeability and conductivity, respectively.

The cross-sections of the sheets in the $(x, y)$-plane all have a finite thickness $2 h$. However, we will approximate the sheets by infinitely thin ones. This is allowed if the penetration depth $\delta$ of the surface current in thickness-direction (where $\delta=\sqrt{2 / \mu \sigma \omega}$, see [7], Sect. 46, or [2]) is large compared to half the thickness of the sheet, i.e. if

$$
\frac{h}{\delta}=\sqrt{\frac{h^{2}}{2} \mu \sigma \omega} \ll 1,
$$

so, for not too large values of $\omega$.

Typical for a copper sheet, as we wish to consider here, are the following numerical values:

$$
2 h=2.5 * 10^{-3} \mathrm{~m}, \quad \mu=4 \pi * 10^{-7} \mathrm{H} / \mathrm{m}, \quad \sigma=5.8 * 10^{7} 1 / \mathrm{Ohm} \cdot \mathrm{m} .
$$

For frequencies of the order $\omega=10^{3} \mathrm{rad} / \mathrm{sec}$, or less, this yields

$$
\delta \geq 5.21 * 10^{-3} \mathrm{~m}
$$

which is large enough to justify the approximation leading to an infinitely thin sheet, which will be called a strip subsequently (it can be shown that by this approximation we introduce an error of at most 3 percent, see [2]).

In the model of a strip, we replace the current density $\mathbf{J}$ (in $\mathrm{Amp} / \mathrm{m}^{2}$ ) by the current per unit of length in the width-direction $\mathbf{j}$ (in Amp/m), defined as

$$
\mathbf{j}=\int_{-h}^{h} \mathbf{J} \mathrm{d} y .
$$

Since we consider sets of $N$ individual strips, we denote the current in the $p^{\text {th }}$ strip $\left(\mathbf{x} \in S_{p}\right.$, conductivity $\sigma=\sigma_{p}$ ) by $\mathbf{j}_{p}$.

The approximation to strips simplifies the boundary conditions considerably, in so far that we now have to consider conditions at the plane $y=0$ only. At $y=0$, there will be jumps in $\mathbf{H}$ and $\mathbf{E}$ across the surface $\mathcal{S}$, the joint surface of the strips, having its normal vector in the $y$-direction $\left(\mathbf{n}=\mathbf{e}_{y}\right)$. Denoting the jump across $\mathcal{S}$ by $\llbracket \rrbracket$, we have

$$
\llbracket \mathbf{E} \times \mathbf{n} \rrbracket=\llbracket \mathbf{H} \cdot \mathbf{n} \rrbracket=0, \quad \llbracket \mathbf{E} \cdot \mathbf{n} \rrbracket=Q_{s}, \quad \llbracket \mathbf{H} \times \mathbf{n} \rrbracket=-\mathbf{K}_{s},
$$


where $Q_{s}$ and $\mathbf{K}_{s}$ are surface charge and surface current density, respectively. For our purposes $Q_{s}$ is irrelevant, whereas $\mathbf{K}_{s}$ is equal to

$$
\mathbf{K}_{s}=\mathbf{j}_{p}, \quad \mathbf{x} \in \mathcal{S}_{p}, \quad p=1,2, \ldots, N .
$$

The set of equations and boundary conditions formulated above for a set of infinitely long strips allows a solution that is independent of the longitudinal $z$-direction. As a consequence, the current $\mathbf{j}_{p}$ has a component in the $z$-direction depending on $x$ only, i.e.

$$
\mathbf{j}_{p}=j_{p}(x) \mathbf{e}_{z}, \quad x \in\left[a_{p}, b_{p}\right] .
$$

As a direct consequence of (10), we find that the only non-zero components of $\mathbf{E}$ and $\mathbf{H}$ are

$$
\left\{E_{z}, H_{x}, H_{y}\right\}
$$

From now on, we restrict ourselves to the upper half plane $\{(x, y) \mid y>0\}$, where the following equations hold for $E_{z}=E(x, y), H_{x(y)}=H_{x(y)}(x, y)$ :

$$
\begin{gathered}
\frac{\partial E}{\partial x}=-i \mu \omega H_{y}, \quad \frac{\partial E}{\partial y}=i \mu \omega H_{x}, \\
\frac{\partial H_{y}}{\partial x}-\frac{\partial H_{x}}{\partial y}=0,
\end{gathered}
$$

together with the boundary conditions at $y=0$,

$$
\begin{aligned}
& \text { for } x \in S \\
& \qquad E(x, 0)=\frac{1}{2 \sigma h} j(x), \quad H_{x}(x, 0)=-\frac{1}{2} j(x),
\end{aligned}
$$

for $x \notin S$

$$
\frac{\partial E}{\partial y}(x, 0)=0, \quad H_{x}(x, 0)=0,
$$

where $j(x)=j_{p}(x)$, for $x \in S_{p}$. Here, $S$ and $S_{p}$ are the two-dimensional representations (the cross-sections in the $(x, y)$-plane) of $\mathcal{S}$ and $\mathcal{S}_{p}$. Note that in the first boundary condition of (13) the right-hand side is the $E$-field inside the sheet according to Ohm's law, and that the factor $1 / 2$ in the second condition is due to the fact that $H_{x}$ just below the strip $\left(y=0^{-}\right)$is equal but opposite to $H_{x}$ just above the strip $\left(y=0^{+}\right)$. The first condition of (14) is due to symmetry.

These conditions must still be complemented by Sommerfeld's radiation condition, stating that

$$
\nabla E \rightarrow \mathbf{0}, \quad H_{x(y)} \rightarrow 0, \quad \text { for } \sqrt{x^{2}+y^{2}} \rightarrow \infty .
$$

From the relations (11) and (12) it follows directly that $H_{x}, H_{y}$ and $E$ all satisfy the Laplace equation in the half-space $y>0$. For $H_{x}$ this leads us to the following boundary value problem

$$
\begin{array}{rlrl}
\Delta H_{x}(x, y) & =0, & \text { on } & \{(x, y) \mid y>0\}, \\
H_{x}(x, 0) & =-\frac{1}{2} j(x), & & x \in S, \\
H_{x}(x, 0) & =0, & x \notin S,
\end{array}
$$




$$
H_{x}(x, y) \rightarrow 0, \quad \sqrt{x^{2}+y^{2}} \rightarrow \infty .
$$

The solution of this problem has the integral representation

$$
H_{x}=-\frac{1}{2 \pi} \int_{S} j(\xi) \frac{y}{y^{2}+(x-\xi)^{2}} \mathrm{~d} \xi
$$

From this result, the following expression for the $y$-component of the magnetic field can be derived:

$$
H_{y}=\frac{1}{2 \pi} \int_{S} j(\xi) \frac{x-\xi}{y^{2}+(x-\xi)^{2}} \mathrm{~d} \xi
$$

For the electric field $E(x, y)$, we assume that this field is built up from a scattered field $E^{s c}(x, y)$, due to the current $j(x)$, plus a bias field $E^{b}(x, y)$, generating these currents. So

$$
E(x, y)=E^{s c}(x, y)+E^{b}(x, y) .
$$

Since only the total field is determined, this splitting up is rather arbitrary. However, once $E^{b}(x, y)$ is chosen, $E^{s c}(x, y)$ is completely determined by the Maxwell equations and boundary conditions. We will choose $E^{b}(x, y)$ such that it satisfies the Maxwell equations (11) and (12), the boundary condition (14), and the radiation condition (15). The bias field is supposed to act in free space without any conductor. Then, $E^{s c}(x, y)$ is coupled to $E^{b}(x, y)$ by boundary condition (13), yielding

$$
E(x, 0)=E^{s c}(x, 0)+E^{b}(x, 0)=\frac{1}{2 h \sigma(x)} j(x), \quad \text { for } \quad x \in S .
$$

For the scattered field, the first radiation condition is replaced by

$$
E^{s c} \rightarrow 0, \quad \text { for } \sqrt{x^{2}+y^{2}} \rightarrow \infty .
$$

We now choose the bias field such that $E^{b}(x, 0)$ is uniform on each strip and zero outside the strips $(x \notin S)$. Moreover, we assume that the strips are coupled in groups of one or more strips, where each group is connected to one source. This means that for each group, $E^{b}(x, 0)$ has the same constant value on each strip of this group. For the notation, we assume that we have $L$ different groups consisting of one or more strips, total partial surface $S_{l}$, and such that $S=\bigcup_{l=1}^{L} S_{l}$.

On each group, we express $E^{b}(x, 0)$ by characteristic functions $\psi_{l}(x)=\mathbf{1}_{\left[S_{l}\right]}, l=1, \ldots, L$, being zero everywhere except on the strips of group $S_{l}$, where it has the value 1 . Denoting the constant value of $E^{b}(x, 0)$ on $S_{l}$ by $C_{l}$, we have that $E^{b}(x, 0)$ can be written as

$$
E^{b}(x, 0)=\sum_{l=1}^{L} C_{l} \psi_{l}(x) .
$$

For the inner product of two characteristic functions $\psi_{l}$ and $\psi_{l^{\prime}}$, where $l, l^{\prime} \in\{1, \ldots, L\}$, one has

$$
\left(\psi_{l}, \psi_{l^{\prime}}\right)=\int_{-\infty}^{\infty} \psi_{l}(x) \psi_{l^{\prime}}(x) \mathrm{d} x=D_{l} \delta_{l l^{\prime}}
$$

where $\delta_{l l^{\prime}}$ is is the Kronecker delta function, which is equal to one if $l=l^{\prime}$ and zero if $l \neq l^{\prime}$, and $D_{l}$ is the sum of the widths of all strips of group $l$. 
To obtain a well-defined problem description, we still need $L$ extra relations to determine the the $L$ as yet unknown constants $C_{1}, \ldots, C_{L}$. We require that the total current in each group of strips is prescribed by

$$
\int_{S_{l}} j(x) \mathrm{d} x=\int_{-\infty}^{\infty} j(x) \psi_{l}(x) \mathrm{d} x=I_{l}, \quad \text { for } \quad l=1, \ldots, L .
$$

Note here that we are not at all interested in the specific value for the bias field for all $(x, y)$; we are mainly focussing on the current distribution $j(x)$ and the resulting magnetic field $\mathbf{H}(x, y)$ outside the strips.

Introducing a typical length scale $D$ and a typical current density scale $j^{*}$, we can write our equations in a dimensionless form (without changing the notations):

for $y>0$

$$
\begin{gathered}
\frac{\partial E}{\partial x}=-i H_{y}, \quad \frac{\partial E}{\partial y}=i H_{x} \\
\frac{\partial H_{y}}{\partial x}-\frac{\partial H_{x}}{\partial y}=0
\end{gathered}
$$

for $x \in S$

$$
E^{s c}(x, 0)=\varepsilon j(x)-\sum_{l=1}^{L} c_{l} \psi_{l}(x), \quad H_{x}(x, 0)=-\frac{1}{2} j(x)
$$

for $x \notin S$

$$
\frac{\partial E}{\partial y}(x, 0)=0, \quad H_{x}(x, 0)=0
$$

and

$$
\int_{-\infty}^{\infty} j(x) \psi_{l}(x) \mathrm{d} x=\frac{I_{l}}{D j^{*}}
$$

where

$$
\varepsilon=\frac{1}{2 h \sigma \mu \omega D}, \quad c_{l}=\frac{C_{l}}{\mu \omega D j^{*}}, \quad j^{*}=\frac{\sum_{l=1}^{L} I_{l}}{\sum_{l=1}^{L} D_{l}} .
$$

Note that in dimensionless form $\left(\psi_{l}, \psi_{l^{\prime}}\right)=\left(D_{l} / D\right) \delta_{l l^{\prime}}$.

For the scattered field, we can derive on using (17) and (18) in (25), and with (21), the following expression

$$
E^{s c}(x, y)=-\frac{i}{4 \pi} \int_{S} j(\xi) \log \left((x-\xi)^{2}+y^{2}\right) \mathrm{d} \xi .
$$

By substituting this expression and (22) into (20), we arrive at the integral equation for $j(x)$

$$
\frac{1}{2 \pi} \int_{S} j(\xi) \log |x-\xi| \mathrm{d} \xi-i \varepsilon j(x)=-i \sum_{l=1}^{L} c_{l} \psi_{l}(x), \quad \text { for } \quad x \in S
$$

This integral equation is a Fredholm equation of the second kind. 
In the case of one strip, reaching from $x=-a$ to $x=a$, we choose $D=a$, and $j^{*}=I / 2 a$. We then obtain the following integral equation:

$$
\frac{1}{2 \pi} \int_{-1}^{1} j(\xi) \log |x-\xi| \mathrm{d} \xi-i \varepsilon j(x)=C, \quad \text { for } \quad x \in[-1,1]
$$

with the unicity condition, to determine the still unknown complex constant $C$,

$$
\int_{-1}^{1} j(x) \mathrm{d} x=2
$$

In the next section, we shall present a general analysis of (33)-(34) and its analytical solution. The general approach to tackle the integral equation (32) for a system of $N$ strips will be presented in Section 4.

\section{General analysis for one strip}

\subsection{General analysis of weakly singular integral equation}

In this section, we show that the current distribution $j(x)$ is twice differentiable inside the strip and that its derivative has a logarithmic singularity in the edge points $x= \pm 1$.

The current satisfies (33), which we write here as

$$
(\mathcal{K}-i \varepsilon) \varphi=f
$$

where $\mathcal{K}$ denotes the integral operator defined on the Hilbert space $L_{2}([-1,1])$ of square integrable functions on $[-1,1]$, given by

$$
(\mathcal{K} \varphi)(x)=\frac{1}{2 \pi} \int_{-1}^{1} \varphi(\xi) \log |x-\xi| \mathrm{d} \xi, \quad x \in[-1,1] .
$$

The weakly singular kernel $\log |x-\xi|$, with $-1<x, \xi<1$, is square integrable and symmetric. Hence, $\mathcal{K}$ is a compact Hermitian operator and (35) has a unique solution for each right-hand side $f \in L_{2}([-1,1])$. Moreover, if $f$ is even/odd, the unicity of the solution of (35) infers that $\varphi$ is even/odd.

The subspace of all $L_{2}$-differentiable functions in $L_{2}([-1,1])$ is denoted by $H_{2,1}([-1,1])$. For $\varphi \in H_{2,1}([-1,1])$, its $L_{2}$-derivative is denoted by $\mathcal{D} \varphi$. With the inner product (see [12, Appendix])

$$
(\varphi, \psi)_{2,1}=(\varphi, \psi)_{L_{2}}+(\mathcal{D} \varphi, \mathcal{D} \psi)_{L_{2}}
$$

the subspace $H_{2,1}([-1,1])$ is a Hilbert space. The functions in $H_{2,1}([-1,1])$ are continuous and satisfy

$$
\max _{x \in[-1,1]}|\varphi(x)| \leq 2 \sqrt{2}\|\varphi\|_{2,1}
$$

Similarly, $H_{2,2}([-1,1])$ consists of all differentiable functions on $[-1,1]$ for which the derivative belongs to $H_{2,1}([-1,1])$. 
According to [13], for $\varphi \in L_{2}([-1,1])$, we have $\mathcal{K} \varphi \in H_{2,1}([-1,1])$ and the $L_{2}$-derivative of $\mathcal{K} \varphi$ is

$$
(\mathcal{D} \mathcal{K} \varphi)(x)=\frac{1}{2 \pi} \int_{-1}^{1} \frac{\varphi(\xi)}{x-\xi} \mathrm{d} \xi, \quad-1<x<1 .
$$

Given $f \in H_{2,1}(\mathbb{R}),(35)$ can be written as

$$
(\mathcal{D} \mathcal{K} \varphi)(x)=\frac{1}{2 \pi} \int_{-1}^{1} \frac{\varphi(\xi)}{x-\xi} \mathrm{d} \xi=i \varepsilon \mathcal{D} \varphi+\mathcal{D} f,
$$

where $\varphi \in H_{2,1}(\mathbb{R})$, since $\mathcal{K} \in H_{2,1}(\mathbb{R}), \varphi, f \in H_{2,1}(\mathbb{R})$, and $\varphi=(\mathcal{K} \varphi-f) / i \varepsilon$.

A straightforward manipulation, based on integration by parts results in the relation,

$$
\mathcal{D K} \varphi=\mathcal{K} \mathcal{D} \varphi+\frac{1}{2 \pi}[\varphi(-1) \log (1+x)-\varphi(1) \log (1-x)] .
$$

We see that $\mathcal{K} \varphi \in H_{2,2}([-1,1])$ and $f \in H_{2,2}([-1,1])$, if and only if $\varphi(-1)=\varphi(1)=0$.

Using (41) in (40), we obtain

$$
\mathcal{D} \varphi=\frac{1}{i \varepsilon}\left[\mathcal{K} \mathcal{D} \varphi+\frac{1}{2 \pi}[\varphi(-1) \log (1+x)-\varphi(1) \log (1-x)]-\mathcal{D} f\right] .
$$

Next, we shall derive the asymptotic behaviour of the solution $\varphi$ at the edges $x= \pm 1$. To this end, we introduce the operator $\mathcal{T}$ from $L_{2}([-1,1])$ into $H_{2,1}([-1,1])$ by

$$
(\mathcal{T} \psi)(x)=\int_{0}^{x} \psi(\xi) \mathrm{d} \xi
$$

By applying $\mathcal{T}$ on (42), we conclude that

$$
\varphi=\frac{1}{2 \pi i \varepsilon}[\varphi(1)(1-x) \log (1-x)+\varphi(-1)(1+x) \log (1+x)]+F,
$$

where

$$
F=\frac{1}{2 \pi i \varepsilon}[(\varphi(1)-\varphi(-1)) x+2 \pi(\mathcal{T} \mathcal{K} \mathcal{D} \varphi-f)]+c,
$$

with $c$ a constant and $F \in H_{2,2}([-1,1])$.

From this, we infer that equation (33) for the current distribution has a solution that is even, $j(x)=j(-x)$, having edge behaviour according to

$$
j(x)=\frac{1}{2 \pi i \varepsilon} j(1)[(1-x) \log (1-x)+(1+x) \log (1+x)]+\tilde{F},
$$

where $\tilde{F} \in H_{2,2}([-1,1])$. 


\subsection{Solution in terms of Legendre polynomials}

In Section 2, we derived the integral equation for the current distribution in one strip, while in the previous section some qualitative results of the solution of this equation have been presented. We have seen which kind of functions we can expect as a solution, the behaviour of the solution at the edges, and some symmetry properties. In this section, taking into account all these properties, we present the solution in terms of Legendre polynomials; see [14].

We start from the Fredholm equation of second kind for the complex current $j$, given by (33), with the unicity condition (34). The latter condition uniquely determines the constant $C$.

From the preceding analyses, we obtain that the solution $j$ satisfies (46) and that $j$ is even. According to (40),

$$
\frac{1}{2 \pi} \int_{-1}^{1} \frac{j(\xi)}{x-\xi} \mathrm{d} \xi=i \varepsilon j^{\prime}(x), \quad x \in(-1,1) .
$$

In order to determine $j$, we expand $j$ in terms of Legendre polynomials $P_{n}$, by writing

$$
j(x)=\sum_{k=0}^{\infty} J_{k} P_{2 k}(x),
$$

where we used that $j$ is even.

Using the condition (34), and the orthogonality of the Legendre polynomials, we obtain $J_{0}=1$.

With

$$
Q_{n}(x)=\frac{1}{2} \int_{-1}^{1} \frac{P_{n}(\xi)}{x-\xi} \mathrm{d} \xi, \quad x \in[-1,1],
$$

where the functions $Q_{n}, n=0,1,2, \ldots$, are Legendre functions of the second kind, the integral equation (47) with (48) substituted into it, yields

$$
\sum_{k=0}^{\infty} J_{k} Q_{2 k}=i \pi \varepsilon \sum_{k=1}^{\infty} J_{k} P_{2 k}^{\prime}
$$

Multiplying (50) by $P_{2 l-1}(x)$, integrating the result with respect to $x$ from $x=-1$ to 1 , and using the relations (see $[14, \mathbf{8 . 1 4 . 8}]$ )

$$
\int_{-1}^{1} Q_{2 k}(x) P_{2 l-1}(x) \mathrm{d} x=\frac{1}{(2 l-2 k-1)(k+l)},
$$

and

$$
\int_{-1}^{1} P_{2 k}^{\prime}(x) P_{2 l-1}(x) \mathrm{d} x= \begin{cases}2, & k \geq l \\ 0, & k<l\end{cases}
$$

we arrive at

$$
2 \pi i \varepsilon \sum_{k=l}^{\infty} J_{k}-\sum_{k=1}^{\infty} a_{l k} J_{k}=\frac{1}{l(2 l-1)}
$$


where

$$
a_{l k}=\frac{1}{(2 l-2 k-1)(k+l)}, \quad k, l=1,2, \ldots
$$

We conclude that the analytical expression of the solution for the looked-for current distribution $j(x)$ in one strip can be presented by (48), where the coefficients $J_{k}$ follow from the infinite linear system of equations (53). Note that here $j(x)$ is the dimensionless current density; to obtain the dimensional one, we have to multiply the $j(x)$ found here by $j^{*}=I / 2 a$.

\subsection{External magnetic field}

In this section, we calculate the external magnetic field due to the current in one infinitely long strip.

In (17) and (18), we have represented the external magnetic field components $H_{x}$ and $H_{y}$ as integrals on $j(x)$. To evaluate these representations, we first write

$$
\frac{1}{\pi} \frac{y}{y^{2}+(x-\xi)^{2}}=\frac{1}{2 \pi i}\left(\frac{1}{\xi-z}-\frac{1}{\xi-\bar{z}}\right)
$$

and

$$
-\frac{1}{\pi} \frac{x-\xi}{y^{2}+(x-\xi)^{2}}=\frac{1}{2 \pi}\left(\frac{1}{\xi-z}+\frac{1}{\xi-\bar{z}}\right),
$$

where $z=x+i y$. Next, we define the linear mapping (Hilbert transformation)

$$
\mathbb{H} j(z)=\frac{1}{2 \pi i} \int_{-1}^{1} \frac{j(\xi)}{z-\xi} \mathrm{d} \xi, \quad z \in \mathbb{C}, \quad \operatorname{Im} z>0 .
$$

Then, the components of the magnetic field can be presented in terms of $\mathbb{H} j$ as

$$
H_{x}=\frac{1}{2}((\mathbb{H} j)(z)-(\mathbb{H} j)(\bar{z}))
$$

and

$$
H_{y}=\frac{i}{2}((\mathbb{H} j)(z)+(\mathbb{H} j)(\bar{z})) .
$$

From the expression (48) for the current, we obtain

$$
\mathbb{H} j(z)=\sum_{k=0}^{\infty} \frac{J_{k}}{2 \pi i} \int_{-1}^{1} \frac{P_{2 k}(\xi)}{z-\xi} \mathrm{d} \xi=\frac{1}{\pi i} \sum_{k=0}^{\infty} J_{k} Q_{2 k}(z),
$$

where we have used (49). Note that the function $Q_{k}(z)$ is analytic on $\mathbb{C} \backslash[-1,1]$; so the mappings

$$
(x, y) \longmapsto Q_{k}(x+i y) \quad \text { and } \quad(x, y) \longmapsto Q_{k}(x-i y)
$$

are harmonic. Moreover, in the interval $[-1,1]$, there is the jump relation (see $[14, \mathbf{8 . 3 . 3}]$ )

$$
\frac{1}{\pi i}\left[Q_{k}(x-i 0)-Q_{k}(x+i 0)\right]=P_{k}(x),
$$

and, finally,

$$
\lim _{|z| \rightarrow \infty} Q_{k}(z)=0 .
$$


Hence, the components of the magnetic field can be written as

$$
H_{x}(x, y)=\frac{1}{2 \pi i} \sum_{k=0}^{\infty} J_{k} Q_{2 k}(x+i y)-\frac{1}{2 \pi i} \sum_{k=0}^{\infty} J_{k} Q_{2 k}(x-i y),
$$

and

$$
H_{y}(x, y)=\frac{1}{2 \pi} \sum_{k=0}^{\infty} J_{k} Q_{2 k}(x+i y)+\frac{1}{2 \pi} \sum_{k=0}^{\infty} J_{k} Q_{2 k}(x-i y) .
$$

\subsection{Resistance for one sheet}

An important quantity of practical interest is the loss produced by the currents. Therefore, also the Ohmic loss will be expressed in terms of the coefficients $J_{k}$. In a conducting body of volume $V$, the Ohmic loss is given by

$$
\frac{1}{\sigma} \int_{V}|\mathbf{J}|^{2} \mathrm{~d} V
$$

For the infinitely long and thin sheet we consider, the Ohmic loss $P$ per unit of length (in Watt $/ \mathrm{m}$ ) is found by replacing the integral over $V$ by one over the cross-sectional surface $S$ and, moreover, by using for $\mathbf{J}$

$$
\mathbf{J}=\frac{1}{2 h} \frac{I}{2 a} j(x) \mathbf{e}_{z},
$$

where $j(x)$ is dimensionless. This yields

$$
P=\frac{I^{2}}{8 \sigma h a} \int_{-1}^{1}|j(x)|^{2} \mathrm{~d} x
$$

Besides this, the power dissipated per unit length of the conductor, can be expressed in terms of the total current $I$ and the resistance $R$ per unit of length ( $R$ in $\mathrm{Ohm} / \mathrm{m})$, as

$$
P=R I^{2} \text {. }
$$

Combining (66) and (67), we get

$$
R=\frac{1}{8 \sigma h a} \int_{-1}^{1}|j(x)|^{2} \mathrm{~d} x
$$

Using in the latter relation, the expansion (48) and the orthogonality of Legendre polynomials, we finally arrive at

$$
R=\frac{1}{4 \sigma h a} \sum_{k=0}^{\infty} \frac{\left|J_{k}\right|^{2}}{4 k+1}
$$




\section{System of $N$ strips}

In this section, we shall present the solution of the integral equation (32) plus the extra conditions (24). We consider a system of $N$ parallel strips, divided in $L$ groups of mutually connected strips. Each group $l, l \in\{1, \ldots, L\}$, occupies an area $S_{l}$, such that $S=\bigcup_{l=1}^{L} S_{l}$. For convenience, we write (32) in a short-hand notation,

$$
\mathcal{K} j-i \varepsilon j=-i \sum_{l=1}^{L} c_{l} \psi_{l}
$$

where

$$
\mathcal{K} j=(\mathcal{K} j)(x)=\frac{1}{2 \pi} \int_{S} j(\xi) \log |x-\xi| \mathrm{d} \xi .
$$

We recall that $\left(\psi_{l}, \psi_{l^{\prime}}\right)=\left(D_{l} / D\right) \delta_{l l^{\prime}}$, so that the projection $\mathcal{P} j$ of $j$ onto the linear span $\operatorname{lin}\left(\left\{\psi_{l} \mid l=1,2, \ldots, L\right\}\right)$ is given by $([15])$

$$
\mathcal{P} j=\sum_{l=1}^{L} \frac{D}{D_{l}}\left(j, \psi_{l}\right) \psi_{l}=\sum_{l=1}^{L} \tilde{I}_{l} \psi_{l},
$$

according to $(29)$ and with $\tilde{I}_{l}=I_{l} / D_{l} j^{*}$. Writing

$$
j_{\perp}=j-\mathcal{P} j,
$$

and applying the operation $(\mathcal{I}-\mathcal{P})$ to $(70)$, we obtain

$$
(\mathcal{I}-\mathcal{P}) \mathcal{K} j-i \varepsilon j_{\perp}=0
$$

as $\mathcal{P} \psi_{l}=\psi_{l}$. With $j$ according to (73), this yields

$$
(\mathcal{I}-\mathcal{P}) \mathcal{K} j_{\perp}-i \varepsilon j_{\perp}=-\sum_{l=1}^{L} \tilde{I}_{l}(\mathcal{I}-\mathcal{P}) \mathcal{K} \psi_{l} .
$$

Note that at this point, we have eliminated the unknown coefficients $c_{l}$ in favour of the given partial currents $I_{l}$.

The unknown function is now $j_{\perp}$, which we determine using the method of moments; see [16]. We approximate $j_{\perp}$ by a finite series of basis functions $\varphi_{m}$, where the functions $\varphi_{m}$ form an orthogonal set and are in the kernel, or null space, of $\mathcal{P}$, i.e. $\mathcal{P} \varphi_{m}=0$, or

$$
\left(\varphi_{m}, \psi_{l}\right)=0, \quad \text { and } \quad\left(\varphi_{m}, \varphi_{m^{\prime}}\right)=0, \quad \text { for } m \neq m^{\prime}
$$

for all $l \in\{1, \ldots, L\}$, and all $m$.

In analogy with the preceding section, we choose Legendre polynomials for $\varphi_{m}$. However, for a system of $N$ strips, not necessarily of the same width, a scaling to the interval $[-1,1]$, as in Section 3.2, is not possible. Therefore, in the case of $N$ parallel strips we have to introduce shifted and scaled Legendre polynomials $\phi_{k, q}, k=1,2, \ldots, q=1,2, \ldots, N$ by

$$
\phi_{k, q}=P_{k}\left(\frac{x-s_{q}}{d_{q}}\right), \quad \text { for } \quad x \in\left[a_{q}, b_{q}\right]
$$


where $s_{q}=\left(a_{q}+b_{q}\right) / 2, d_{q}=\left(b_{q}-a_{q}\right) / 2, q=1,2, \ldots, N$. Moreover, $\phi_{k, q}=0$, for $x \notin\left[a_{q}, b_{q}\right]$. The shifted and scaled Legendre polynomials are used to build the basis functions. We create a series expansion that is specific for each strip, according to

$$
j_{\perp}=\sum_{k=1}^{K} \sum_{q=1}^{N} \alpha_{k, q} \phi_{k, q}+\sum_{q=1}^{N-L} \alpha_{0, q} \phi_{0, q}=\sum_{m=1}^{M} \alpha_{m} \varphi_{m}(x) .
$$

Here, for $m=1, \ldots, N-L$,

$$
\alpha_{m}=\alpha_{0, q}, \quad \varphi_{m}=\phi_{0, q}, \quad m=q=1, \ldots, N-L,
$$

with $\phi_{0, q}$ to be specified further on, whereas for $m=N-L+1, \ldots, M=(K+1) N-L$,

$$
\alpha_{m}=\alpha_{k, q}, \quad \varphi_{m}=\phi_{k, q}, \quad m=q+k N-L,
$$

for $k=1, \ldots, K$, and $q=1, \ldots, N$. So, $K$ denotes the number of degrees of Legendre functions that is included, and $M$ is the total number of basis functions. The basis functions must be orthogonal with respect to each other as well as to the functions $\psi_{l}$. For $m \in[N-L+1, M]$ these conditions are satisfied because then $\left(m=q+k N-L, n=q^{\prime}+k^{\prime} N-L\right)$

$$
\left(\varphi_{m}, \varphi_{n}\right)=\int_{S} \phi_{k, q}(x) \phi_{k^{\prime}, q^{\prime}}(x) \mathrm{d} x=\frac{2 d_{q}}{2 k+1} \delta_{k k^{\prime}} \delta_{q q^{\prime}}
$$

and

$$
\left(\varphi_{m}, \psi_{l}\right)=\int_{S} \phi_{k, q}(x) \psi_{l}(x) \mathrm{d} x=0
$$

for $q=1, \ldots, N, l=1, \ldots, L$.

According to (73), the total current $j$ is approximated for a part by $L$ piecewise constant functions $\psi_{l}$. So, for $j_{\perp}, N-L$ constant functions are left that can differentiate the current distributions in the $L$ groups. This explains why we split off the sum with the $N-L$ terms in (78). To determine an appropriate choice for the basis functions of degree zero, we first define a basis of characteristic functions $\left\{\mathbf{1}_{\left[a_{1}, b_{1}\right]}, \ldots, \mathbf{1}_{\left[a_{N}, b_{N}\right]}\right\}$. The functions $\psi_{l}$ can be constructed by $N_{l}$ elements of this basis, where $N_{l}$ is the number of strips of group $l$ (clearly $\sum_{l=1}^{L} N_{l}=N$ ). To create the basis functions $\phi_{0, q}$, we must find $N_{l}-1$ linear combinations of the basis elements for each group $l$, such that each $\phi_{0, q}$ is orthogonal with respect to all $\psi_{l}$ and $\phi_{0, q^{\prime}}$, for $q \neq q^{\prime}$. Each function $\phi_{0, q}$ can thus be expressed in a linear combination of characteristic functions. Let us explain this for an example of 3 strips, in which the first and the second strip form one group $\left(l=1, N_{1}=2\right)$ and the third strip forms the second group $\left(l=2, N_{2}=1\right)$. Then, $L=2$, and

$$
\psi_{1}=\mathbf{1}_{\left[a_{1}, b_{1}\right]}+\mathbf{1}_{\left[a_{2}, b_{2}\right]}, \quad \psi_{2}=\mathbf{1}_{\left[a_{3}, b_{3}\right]},
$$

while $N-L=1$, and

$$
\phi_{0,1}=-\mathbf{1}_{\left[a_{1}, b_{1}\right]}+\frac{d_{1}}{d_{2}} \mathbf{1}_{\left[a_{2}, b_{2}\right]}
$$

On the other hand, for a system of three coupled strips $(N=3, L=1)$ we can choose

$$
\psi_{1}=\mathbf{1}_{\left[a_{1}, b_{1}\right]}+\mathbf{1}_{\left[a_{2}, b_{2}\right]}+\mathbf{1}_{\left[a_{3}, b_{3}\right]},
$$


and

$$
\phi_{0,1}=-\mathbf{1}_{\left[a_{1}, b_{1}\right]}+\frac{d_{1}}{d_{2}} \mathbf{1}_{\left[a_{2}, b_{2}\right]}, \quad \phi_{0,2}=-\frac{1}{2} \mathbf{1}_{\left[a_{1}, b_{1}\right]}-\frac{1}{2} \mathbf{1}_{\left[a_{2}, b_{2}\right]}+\frac{d_{1}+d_{2}}{2 d_{3}} \mathbf{1}_{\left[a_{3}, b_{3}\right]} .
$$

Finally, for 3 separate strips $(N=L=3)$, no $\phi_{0, q}$ 's exist and $\psi_{l}=\mathbf{1}_{\left[a_{l}, b_{l}\right]}$ for $l=1,2,3$.

We now return to (75) into which we substitute (78), to obtain

$$
\sum_{m=1}^{M} \alpha_{m}(\mathcal{I}-\mathcal{P}) \mathcal{K} \varphi_{m}-i \varepsilon \sum_{m=1}^{M} \alpha_{m} \varphi_{m}=-\sum_{l=1}^{L} \tilde{I}_{l}(\mathcal{I}-\mathcal{P}) \mathcal{K} \psi_{l}
$$

Taking the inner product of this equation with $\varphi_{n}$, we find

$$
\sum_{m=1}^{M} \alpha_{m}\left((\mathcal{I}-\mathcal{P}) \mathcal{K} \varphi_{m}, \varphi_{n}\right)-i \varepsilon \sum_{m=1}^{M} \alpha_{m}\left(\varphi_{m}, \varphi_{n}\right)=-\sum_{l=1}^{L} \tilde{I}_{l}\left((\mathcal{I}-\mathcal{P}) \mathcal{K} \psi_{l}, \varphi_{n}\right)
$$

Since $(\mathcal{I}-\mathcal{P})$ is a self-adjoint operator, and as $(\mathcal{I}-\mathcal{P}) \varphi_{n}=\varphi_{n}$, this equation reduces to

$$
\sum_{m=1}^{M} \alpha_{m}\left(\mathcal{K} \varphi_{m}, \varphi_{n}\right)-i \varepsilon \sum_{m=1}^{M} \alpha_{m}\left(\varphi_{m}, \varphi_{n}\right)=-\sum_{l=1}^{L} \tilde{I}_{l}\left(\mathcal{K} \psi_{l}, \varphi_{n}\right)
$$

From this equation, the unknown coefficients $\alpha_{m}$ can be determined.

For convenience, we write (85) in matrix formulation as

$$
(\mathbf{A}-i \varepsilon \mathbf{G}) \mathbf{a}=-\mathbf{B f},
$$

where $\mathbf{a}=\left(\alpha_{1}, \ldots, \alpha_{M}\right)^{T}$ and $\mathbf{f}=\left(\tilde{I}_{1}, \ldots, \tilde{I}_{L}\right)^{T}$ are an $M$-vector and an $L$-vector, respectively, while $\mathbf{A}$ and $\mathbf{G}$ are symmetric $M \times M$-matrices and $\mathbf{B}$ is an $M \times L$-matrix, defined by their entries

$$
A_{n m}=\left(\varphi_{n}, \mathcal{K} \varphi_{m}\right), \quad G_{n m}=\left(\varphi_{n}, \varphi_{m}\right), \quad B_{n l}=\left(\varphi_{n}, \mathcal{K} \psi_{l}\right) .
$$

We then have successively:

1. For $m=q+k N-L>N-L$, and $n=q^{\prime}+k^{\prime} N-L>N-L$,

$$
\begin{aligned}
& A_{n m}=\frac{1}{2 \pi} \int_{a_{q^{\prime}}}^{b_{q^{\prime}}} \phi_{k^{\prime}, q^{\prime}}(x) \int_{a_{q}}^{b_{q}} \phi_{k, q}(\xi) \log |x-\xi| \mathrm{d} \xi \mathrm{d} x \\
= & \frac{d_{q^{\prime}} d_{q}}{2 \pi} \int_{-1}^{1} \int_{-1}^{1} P_{k^{\prime}}(x) P_{k}(\xi) \log \left|d_{q^{\prime}} x-d_{q} \xi+s_{q^{\prime}}-s_{q}\right| \mathrm{d} \xi \mathrm{d} x,
\end{aligned}
$$

where we have used the substitutions

$$
x \rightarrow d_{q^{\prime}} x+s_{q^{\prime}}, \quad \text { and } \quad \xi \rightarrow d_{q} \xi+s_{q} .
$$

The kernel of this integral has a logarithmic singularity if and only if $q=q^{\prime}$; otherwise the integrand is regular, and the integral can simply be calculated numerically. In case 
$q=q^{\prime}$, we obtain

$$
\begin{aligned}
A_{n m} & =\frac{d_{q}^{2}}{2 \pi} \int_{-1}^{1} \int_{-1}^{1} P_{k^{\prime}}(x) P_{k}(\xi) \log \left(d_{q}|x-\xi|\right) \mathrm{d} \xi \mathrm{d} x \\
& = \begin{cases}\frac{8 d_{q}^{2}}{2 \pi\left(k+k^{\prime}\right)\left(k+k^{\prime}+2\right)\left[\left(k-k^{\prime}\right)^{2}-1\right]}, & \text { if } k+k^{\prime} \text { even }, \\
0, & \text { if } k+k^{\prime} \text { odd. }\end{cases}
\end{aligned}
$$

If $k=0$, or $m=q \leq N-L$, we have $\varphi_{m}=\phi_{0, q}$, which is either constant on a strip or zero. We evaluate $A_{n m}$ for the example of $\varphi_{m}=\mathbf{1}_{\left[a_{q}, b_{q}\right]}$. Note that $\phi_{0, q}$ is a linear combination of characteristic functions, so the separate contributions can be superposed with the corresponding coefficients (as explained before). We obtain for $A_{n m}$ :

$$
\frac{d_{q^{\prime}} d_{q}}{2 \pi} \int_{-1}^{1} \int_{-1}^{1} P_{k^{\prime}}(x) \log \left|d_{q^{\prime}} x-d_{q} \xi+s_{q^{\prime}}-s_{q}\right| \mathrm{d} \xi \mathrm{d} x .
$$

For $q \neq q^{\prime}$, the integrand is regular and the integral will be calculated numerically; if $q=q^{\prime}$, we use the analytical result

$$
\begin{aligned}
& \frac{d_{q}^{2}}{2 \pi} \int_{-1}^{1} \int_{-1}^{1} P_{k^{\prime}}(x) \log \left(d_{q}|x-\xi|\right) \mathrm{d} \xi \mathrm{d} x \\
= & \begin{cases}\frac{8 d_{q}^{2}}{2 \pi k^{\prime}\left(k^{\prime}+2\right)\left(k^{\prime 2}-1\right)}, & \text { if } k^{\prime} \text { even }, \\
0, & \text { if } k^{\prime} \text { odd }, \\
\frac{d_{q}^{2}}{2 \pi}\left(4 \log 2+4 \log d_{q}-6\right), & \text { if } k^{\prime}=0 .\end{cases}
\end{aligned}
$$

2. The Gram-matrix $\mathbf{G}$ is diagonal due to the orthogonality of the basis functions $\varphi_{m}$ on $S$. For $m>N-L$, we have, for $k=1, \ldots, K$,

$$
G_{m m}=\left(\varphi_{m}, \varphi_{m}\right)=\int_{a_{q}}^{b_{q}} \phi_{k, q}^{2}(x) \mathrm{d} x=d_{q} \int_{-1}^{1} P_{k}^{2}(x) \mathrm{d} x=\frac{2 d_{q}}{2 k+1} .
$$

For $m=n \leq N-L$, the integrand of $G_{m m}$ is constant and the evaluation of the integral trivial.

3. We evaluate the matrix $\mathbf{B}$ for the example of $\psi_{l}=\mathbf{1}_{\left[a_{q}, b_{q}\right]}$ (if $\psi_{l}$ is a linear combination of characteristic functions, the separate contributions can directly be superposed) and for $n>N-L$, to obtain

$$
\begin{aligned}
B_{n l} & =\left(\mathcal{K} \psi_{l}, \varphi_{n}\right)=\frac{1}{2 \pi} \int_{a_{q^{\prime}}}^{b_{q^{\prime}}} \phi_{k^{\prime}, q^{\prime}}(x) \int_{a_{q}}^{b_{q}} \mathbf{1}_{\left[a_{q}, b_{q}\right]} \log |x-\xi| \mathrm{d} \xi \mathrm{d} x \\
& =\frac{d_{q^{\prime}} d_{q}}{2 \pi} \int_{-1}^{1} \int_{-1}^{1} P_{k^{\prime}}(x) \log \left|d_{q^{\prime}} x-d_{q} \xi+s_{q^{\prime}}-s_{q}\right| \mathrm{d} \xi \mathrm{d} x
\end{aligned}
$$


This integral is identical to the one in (90).

\section{$5 \quad$ Numerical results}

In this section, we present the results of the numerical simulations of four different configurations. First, we consider one strip. Second, we consider three examples of systems of three strips:

1. Three strips, each strip connected to a separate source.

2. Three strips connected to one and the same source.

3. Three strips, the first and second of which are connected to one source, and the third to another.

In all situations, we consider strips of the same width and thickness, and of the same material. The values are shown in Table 1. Parameters that vary are the distances between the strips and the frequencies.

Table 1: Values of geometrical and material parameters.

\begin{tabular}{clll}
\hline Symbol & Name & Value & \\
\hline $2 h$ & Thickness of strip & $2.5 \cdot 10^{-3}$ & $\mathrm{~m}$ \\
\hline $2 D$ & Width of strip & $4.0 \cdot 10^{-2}$ & $\mathrm{~m}$ \\
\hline$\sigma$ & Electric conductivity & $5.8 \cdot 10^{7}$ & $\Omega^{-1} \mathrm{~m}^{-1}$ \\
\hline$\mu$ & Magnetic permeability & $4 \pi \cdot 10^{-7}$ & $\mathrm{Hm}^{-1}$ \\
\hline
\end{tabular}

\section{$5.1 \quad$ One strip}

We consider the case of one strip positioned at $[-D, D]$. The total current flowing through the strip is prescribed. The current is expanded in terms of Legendre polynomials, as shown in (48), and the coefficients are calculated from (53).

Figure 2 shows the amplitude of the (dimensionless) current distribution for the four frequencies $\omega=100,400,700,1000 \mathrm{rad} / \mathrm{s}$. We observe that the current at the edges is higher than in the center. This edge-effect, which is due to the self-inductance of the strip, becomes stronger as the frequency increases. For low frequencies, the current is distributed almost uniformly, whereas for high frequencies, the current is more concentrated at the edges. The integrated current is always equal to 2 , in accordance with (34).

To obtain the time-dependent result for the currents, we multiply the amplitude of the current by $\mathrm{e}^{-i \omega t}$. Simulating this case, we observe a phase difference in the system. In Figure 3, the phase difference is shown as function of $x / D$, for the four frequencies $\omega=$ $100,400,700,1000 \mathrm{rad} / \mathrm{s}$. We see that for each frequency only two points are in phase with the source (although the figures insinuate the contrary, these points do not coincide for all frequencies). The currents at the edges are ahead in phase with respect to the source, whereas currents at the center are behind in phase. The phase difference increases with the frequency. 


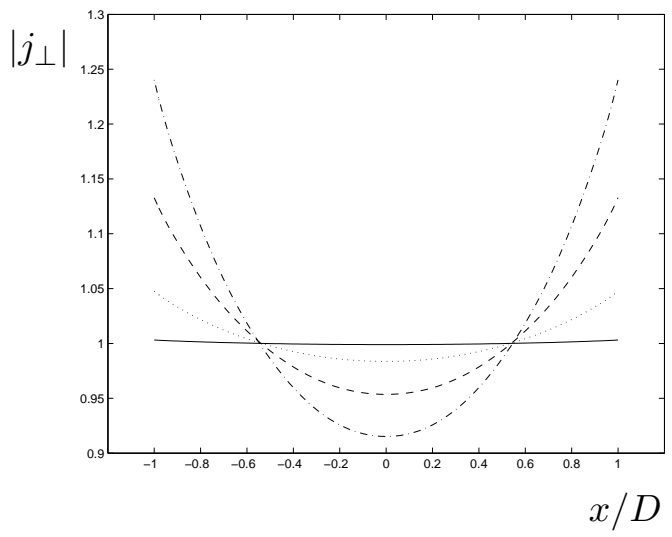

Figure 2: Amplitude of the dimensionless current distribution of one strip, for four frequencies: $\omega=100$ (line), 400(dotted), 700(dashed), 1000(dash-dotted) $\mathrm{rad} / \mathrm{s}$.

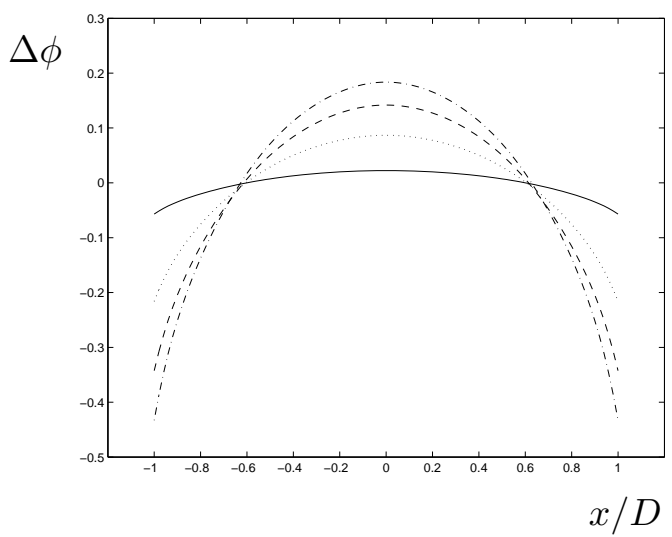

Figure 3: Phase difference of the dimensionless current distribution of one strip, for four frequencies: $\omega=100$ (line), 400(dotted), 700(dashed), 1000(dash-dotted) $\mathrm{rad} / \mathrm{s}$. 


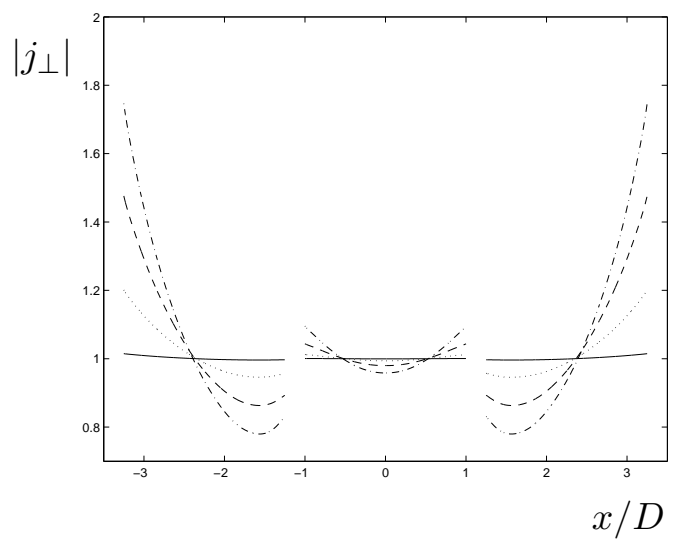

Figure 4: Amplitude of the current distribution of a set of three strips forming three groups, for four frequencies: $\omega=100$ (line), 400(dotted), 700(dashed), 1000(dash-dotted) $\mathrm{rad} / \mathrm{s}$.

\subsection{Configuration of three strips}

We saw in the previous section that an edge-effect occurs in one strip due to the self-inductance of the strip. In a configuration of more strips also mutual inductances play a role. In this section, we consider three situations for a set of three strips: forming three groups, one group and two groups, respectively. We investigate how the current distribution, and specifically the edge-effect, depends on the frequencies and on the distance between the strips.

\subsubsection{Three strips forming three groups}

We consider the case of a strip, positioned at $[-D, D]$, accompanied on both sides by a strip of the same width, both at a distance $d$, where for this case $d=0.25$ (dimensionless). The currents through the three strips are driven by three separate sources, so no Legendre functions of degree zero are needed here. On each strip the same total current is prescribed.

In Figure 4, the amplitude of the current distribution for the set of three strips is shown, for the four frequencies $\omega=100,400,700,1000 \mathrm{rad} / \mathrm{s}$. We again observe the edge-effects for each strip, but the shapes are quite different: the system tries to enforce a global edge-effect. Note that higher frequencies cause stronger edge-effects. Moreover, it is clear that the prescribed total current is the same through all three strips.

Next, we investigate the effect of the distance between the strips on the edge-effect. To this end, we vary the distance between the strips, keeping the widths of the strips the same, and keeping the frequency fixed at $\omega=700 \mathrm{rad} / \mathrm{s}$. We simulate the current distribution for a number of distances $d$ in the range $\left[10^{-2}, 10^{2}\right]$, and register the relative values of the currents at the points $x=a_{1}, b_{1}$ and $a_{2}$; see Figure 1 . The result is shown in Figure 5 , where on the horizontal axis a logarithmic scale for $d$ is used. Note that for small distances $d$ the value of the current in $b_{1}$ tends to the value of the current in $a_{2}$. For larger distances $d$, all values tend to 0.135 , which is the same value as at the edge of one strip, see Figure 2 (absolute value is 1.135). So, the distance has become too large to 'feel' the presence of another strip. 


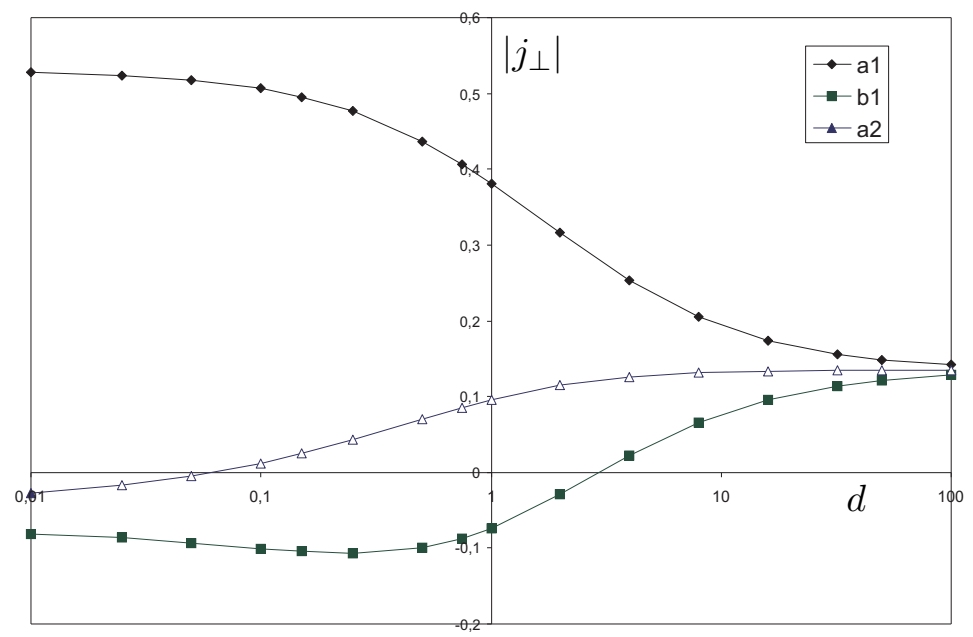

Figure 5: Relative value of the current at the points $x=a_{1}, b_{1}, a_{2}$ for sets of three strips forming three groups, plotted against the distance $d$ between two neighbouring strips (logarithmic scale.

\subsubsection{Three strips forming one group}

Next, we consider a set of three strips connected to one source. In this case, the total current through the whole set of three strips is prescribed (and equal to three times the prescribed current for one strip in the preceding case). This implies that the individual total current through each strip is free, as can be seen from Figure 6. This figure shows the current distributions for four frequencies $\omega=100,400,700,1000 \mathrm{rad} / \mathrm{s}$. The distance between the strips is the same as for the case depicted in Figure 4.

We see that the total current through the central strip is smaller than that through the two side strips. This effect is stronger for higher frequencies. Moreover, the edge-effects at the outer edges of the set are amplified with respect to the preceding case. This can be concluded from a comparison of Figure 6 with Figure 4. As before, the edge-effect increases with increasing frequencies.

\subsubsection{Three strips forming two groups}

As a third and final example, we consider a set of three strips connected to two sources. The configuration is the same as in the preceding two subsections. The first two strips are connected to one source, the prescribed total current being equal to twice the total current through one strip for the first case. The third strip is connected to a separate source and the prescribed total current is here equal to the total current through one strip for the first case.

The situation is now no longer symmetric as can be clearly seen from Figure 7 . In this figure, the current distributions are depicted for the four frequencies $\omega=100,400,700,1000$ $\mathrm{rad} / \mathrm{s}$. The edge-effect is here strongest at the outer edge of the first strip, and this edge-effect is stronger than in the preceding two cases. Again, the edge-effect increases with increasing frequency. 


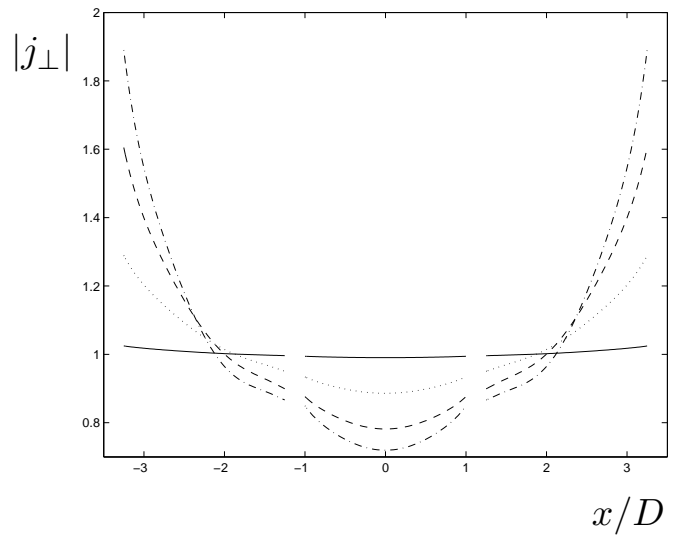

Figure 6: Amplitude of the current distribution of a set of three strips forming one group, for four frequencies: $\omega=100$ (line), 400(dotted), 700(dashed), 1000(dash-dotted) $\mathrm{rad} / \mathrm{s}$.

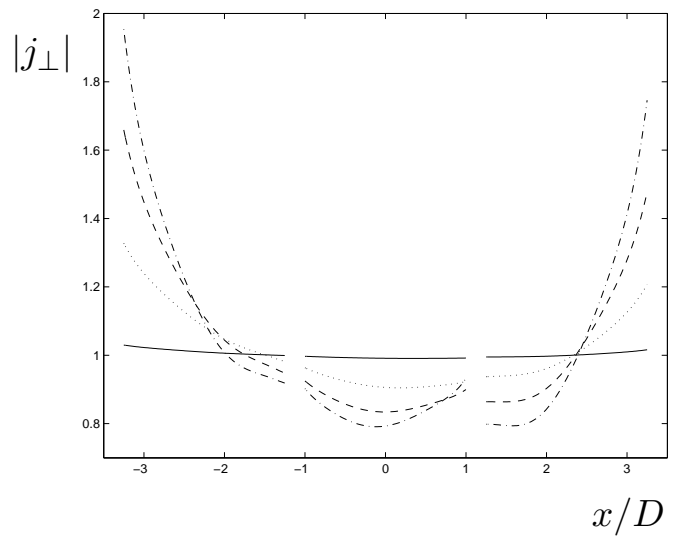

Figure 7: Amplitude of the current distribution of a set of three strips forming two groups, for four frequencies: $\omega=100$ (line), 400(dotted), 700(dashed), 1000(dash-dotted) $\mathrm{rad} / \mathrm{s}$. 


\section{Conclusions}

In this article, the current distribution in a parallel set of conducting strips has been investigated. Assumed is that the strips are negligibly thin and infinitely long, that all the media are non-polarizable and non-magnetizable, and that the electric conductivity $\sigma$ is constant throughout the strips. The current is driven by a biased electric field, which is time harmonic, with a low frequency (in the order of $10^{3} \mathrm{~Hz}$ ). Due to the restricted frequency range, a quasistatic approach can be applied. For the uniqueness of the solution, the current through each group of mutually connected strips is prescribed.

It has been shown that the current only has a component in the longitudinal $z$-direction and only depends on the transversal direction $x$. Moreover, the current distribution must satisfy a Fredholm integral equation of the second kind, containing a logarithmic kernel function. This integral equation has been solved by using a series expansion of the current distribution in terms of Legendre polynomials (the basis functions). The method of moments has been applied with the same basis functions used as test functions.

For one strip the current distribution could be determined completely by analytical means, and consequently also the magnetic field, the resistance, and the Ohmic loss. The results showed the occurrence of edge-effects, and how these effects become stronger with increasing frequency. The time dependence resulted in a phase difference between the current and the applied voltage; this phase difference increases with frequency. The behaviour of the current at the edges of the strip has been determined explicitly, and it has been shown that its derivative has a logarithmic singularity at the edge points.

Also in a system of a finite number of strips, the Legendre polynomials are useful to determine the current distribution by analytical means. The interaction of two different strips yielded a regular integral, which could easily be computed numerically. Not only edge-effects within one strip were found, but also a global edge-effect in the whole system, depending on the distances between the strips and the frequency.

The model we considered can be extended in the following aspects:

- In the existing model we assumed that the material properties of all strips are the same. This assumption is somehow redundant: different material properties can easily be included in this model.

- The fields in this model are time harmonic. By superposition, we can handle a more general time-dependence after expanding this dependence in Fourier modes.

- Instead of infinitely long strips lying in one plane, we can consider sets of circular loops of strips placed on a circular cylinder. This is more in conformity with the real situation of a gradient coil in an MRI scanner. One of the questions that arises then is in how far the curvature of the strips affects the current distribution. Moreover, in this case it is possible to calculate the magnetic field inside the cylinder, which is an important aspect with regard to an adequate performance of the gradient coil. The current distribution in the width direction of the sheets can again be expressed in terms of Legendre polynomials. This will be the subject of a forthcoming paper [4].

\section{Acknowledgement}

This report is based on a project supported by Philips Medical Systems in Best, The Netherlands, and performed by T. Ulicevic under the supervision of G. Peeren and J. Konijn. 


\section{References}

[1] M.T. Vlaardingerbroek and J.A. den Boer, Magnetic Resonance Imaging. Berlin: Springer (1999) 481pp.

[2] T. Ulicevic, Skin effect in a gradient coil. Eindhoven: Final report of the postgraduate program Mathematics for Industry, Eindhoven University of Technology (2001) 91pp.

[3] J.M.B. Kroot, Current Distribution in a Gradient Coil, Modeled as Circular Loops of Strips. Eindhoven: Final report of the postgraduate program Mathematics for Industry, Eindhoven University of Technology (2002) 59pp.

[4] J.M.B. Kroot, S.J.L. van Eijndhoven, A.A.F. van de Ven, Current distribution in sets of circular loops and islands of conducting strips. Forthcoming.

[5] G.N. Peeren, Stream Function Approach for Determining Optimal Surface Currents. Eindhoven: PhD-Thesis (2003) 196 pp.

[6] D. Tomasi, Stream Function Optimization for Gradient Coil Design, Magnetic Resonance in Medicine 45 (2001) 505-512.

[7] L.D. Landau and E.M. Lifshitz, Electrodynamics of Continuous Media. Oxford: Pergamon Press (1960) 417 pp.

[8] R.E. Collin, Foundations for Microwave Engineering. New York: McGraw-Hill (1992) $924 \mathrm{pp}$.

[9] D.J. Bekers, S.J.L. van Eijndhoven, A.A.F. van de Ven, Modelling and Analysis of a Long Thin Conducting Stripline. Journal of Engineering Mathematics 49 (2004) (article in press).

[10] Y.A. Genenko, A.V. Snezhko, A. Usoskin, Finite magnetic shields effect on the transport current in superconducting strips. Physica C 401 (2004) 236-240.

[11] L.K. Forbes, S. Crozier, D.M. Doddrell, Calculating Current Densities and Fields Produced by Shielded Magnetic Resonance Imaging Probes. SIAM Journal of Applied Mathematics 57 (1997) 401-425.

[12] A. Kirsch, An Introduction to the Mathematical Theory of Inverse Problems. Berlin: Springer (1996) $282 \mathrm{pp}$.

[13] A. Zygmund, Trigonometrical Series. New York: Dover (1955) 329 pp.

[14] M. Abramowitz and I.A. Stegun, Handbook of Mathematical Functions. New York: Dover (1972) 1046 pp.

[15] M.A. Golberg and C.S. Chen, Discrete Projection Methods for Integral Equations. Southampton: Computational Mechanics Publications (1997) 425 pp.

[16] R. F. Harrington, Field Computation by Moment Methods. London: Macmillan (1968) 238 pp. 